\title{
Realización de convenios con el Sector Privado como alternativa para la ampliación de las colocaciones de préstamo
}

Making agreements with the Private Sector as an alternative for the expansion of loan placements

\author{
Jorge M. Figueroa \\ jfigueroar@usmp.pe - Universidad San Martin de Porres, Lima \\ Angel F. Navarro \\ angelnr22@gmail.com - Universidad Nacional Tecnológica de Lima Sur, Lima \\ Julio E. Valero \\ jvalero@untels.edu.pe - Universidad Nacional Tecnológica de Lima Sur, Lima \\ Manuel Hilario \\ fhilariof@ucvvirtual.edu.pe - Universidad César Vallejo, Lima
}

Recibido el 31/08/20 | Aceptado el 18/09/20

DOI: https://doi.org/10.47190/nric.v3i1.127

\section{Resumen}

La investigación se realizó para determinar como el modelo de gestión de préstamos que realiza un banco para el sector público se beneficia económicamente por los convenios con el sector privado y la cantidad de clientes calificados para crédito. La población está conformada por los convenios efectuados con seis empresas privadas, la encuesta se llevó a cabo con la participación de 370 personas de los diferentes centros mineros. Se utilizó tecnologías para el análisis de procesos y explotación del Data Warehouse, para la construcción y análisis de información. Se apreció que en promedio el $80 \%$ de los funcionarios, técnicos y administrativos, obreros y operarios encuestados de las empresas privadas están interesados que el banco estatal realice un convenio con sus empresas para que puedan recibir sus remuneraciones en la amplia red que cuenta el banco.

Palabras claves: Red de cobertura bancaria, Modelo de gestión de préstamo, Data Warehouse.

\begin{abstract}
The research was conducted to determine how a bank's loan management model for the public sector benefits financially from private sector agreements and the number of customers qualified for credit. The population consists of the agreements made with six private companies; the survey was carried out with the participation of 370 people from the different mining centers. Technologies were used for the analysis of processes and exploitation of the Data Warehouse, for the construction and analysis of information. It was appreciated that on average $80 \%$ of the officials, technicians and administrative workers, workers and operators surveyed from private companies are interested in the state bank making an agreement with their companies so that they can receive their remuneration in the bank's coverage network.
\end{abstract}

Keywords: Bank coverage network, Loan management model, Data Warehouse.

Como citar: Figueroa-Jorge M., Navarro-Ángel F., Valero-Julio E., Hilario-Manuel (2020). Realización de convenios con el Sector Privado como alternativa para la ampliación de las colocaciones de préstamo. ÑAWPARISUN - Revista de Investigación Científica, 3(1), 59-64. 


\section{Introducción}

Un tema sobre las condiciones del crecimiento económico en un contexto de mayor globalización económica que involucra una mayor integración financiera es cuán factible resulta inmunizar a los sistemas financieros locales de choques externos, sobre todo dado que la literatura sobre crisis cambiarias y bancarias Kaminski y Reinhart (1999) encuentra que los agregados monetarios como el crédito doméstico están entre los mejores predictores de dichas crisis.

Por otro lado, un estudio sobre la importancia de los sistemas financieros para el crecimiento de la productividad y el desarrollo económico es desarrollado por Levine et al (2003) señalando estos autores que mejores sistemas financieros aumentan la probabilidad de que surjan innovaciones exitosas y por consiguiente aceleran el crecimiento económico.

Tomando como indicador del desarrollo del sistema financiero al ratio Crédito al Sector Privado/PBI, en los trabajos de Calderón y Schmidt-Hebbel (2003), Calderón y Servén (2003) y Blyde y Fernández-Arias (2004) encuentran una asociación directa entre esta variable y la tasa de crecimiento del PBI producto, mientras que similar asociación se encuentra en los trabajos de Easterly, Loayza y Montiel (2007), aunque tomando como variable indicativa del desarrollo financiero al ratio $\mathrm{M} 2 / \mathrm{PBI}$.

El propósito del trabajo es de mantener la sostenibilidad del modelo de colocaciones del banco, dándole la oportunidad de seguir creciendo al buscar ampliar nuevos nichos de colocaciones, siempre focalizado en hacer bancarización con inclusión social y mejorar la calidad de vida de sus clientes.

Ante la saturación del programa de préstamos al sector público obliga al banco a ampliar su oferta de colocaciones incrementando los convenios con el sector privado, los cuales permitirá los préstamos y la cobertura de productos y servicios.

El objetivo es conocer lo que representa ampliar las colocaciones en el sector privado, determinar en qué medida el banco se beneficia económicamente por los convenios con el sector privado y determinar si la ampliación de los préstamos al sector privado aumenta la cantidad de clientes calificados para crédito.

Se pudo apreciar que en promedio el $80 \%$ de los funcionarios, técnicos y administrativos, obreros y operarios encuestados de las empresas privadas están interesados que el banco estatal realice un convenio con sus empresas para que puedan recibir sus remuneraciones en la amplia Red que cuenta el banco.

\section{Materiales y métodos}

La encuesta se realizó a 370 personas de los diferentes centros mineros que han efectuado convenios con el banco, las cuales fueron estratificadas de la siguiente manera:

\begin{tabular}{lcc}
$\begin{array}{l}\text { Tabla 1. } \\
\text { Estratificación de la muestra }\end{array}$ & \\
\hline Participantes & Cantidad & Porcentaje \\
\hline Funcionarios ejecutivos & 88.8 & $24.00 \%$ \\
Técnicos Administrativos & 112.85 & $30.50 \%$ \\
Obreros / Operarios & 168.35 & $45.50 \%$ \\
\hline \multicolumn{1}{c}{ TOTAL } & 370 & $100.00 \%$ \\
\hline
\end{tabular}

Se utilizó el método Likert para lograr cuantificar el grado de interés que tienen los encuestados en que se desarrollen los convenios del banco con el sector privado a cada respuesta de las preguntas realizadas se le asignó un valor numérico. Así, cada encuestado obtiene una puntuación respecto a la afirmación y al final se obtiene su puntuación total sumando las puntuaciones obtenidas en relación con todas las preguntas.

Respecto al interés para realizar convenios para recibir las remuneraciones existe una respuesta favorable.

\begin{tabular}{|c|c|c|c|}
\hline $\mathrm{N}^{\circ}$ & Alternativas & Puntaje & Sustento \\
\hline 01 & SI & 2 & Respuesta favorable \\
\hline 02 & NO & 0 & Respuesta desfavorable \\
\hline 03 & No Sabe - No Responde & 1 & Respuesta medianamente favorable \\
\hline
\end{tabular}

Respecto al interés de contar con un banco cercano a su centro de trabajo y que le brinden las facilidades necesarias para realizar sus operaciones bancarias, se tiene respuesta favorable. 
Tabla 3.

Interés de contar con un banco cercano a su centro de trabajo

\begin{tabular}{cccl}
\hline$N^{\circ}$ & Alternativas & Puntaje & \multicolumn{1}{c}{ Sustento } \\
\hline 01 & SI & 2 & Respuesta favorable \\
02 & NO & 0 & Respuesta desfavorable \\
03 & No Sabe - No Responde & 1 & Respuesta medianamente favorable \\
\hline
\end{tabular}

Respecto al tiempo de demora con el banco para realizar el cobro de sus remuneraciones tiene puntaje alto, que significa más de un día, lo cual genera molestias en los clientes.

\begin{tabular}{lccl}
$\begin{array}{l}\text { Tabla } 4 . \\
\text { El tiempo que demora con el banco para sus remuneraciones }\end{array}$ \\
\hline $\mathrm{N}^{\circ}$ & Alternativas & Puntaje & \multicolumn{1}{c}{ Sustento } \\
\hline 01 & Pocos minutos & 0 & $\begin{array}{l}\text { Respuesta desfavorable } \\
\text { Respuesta medianamente } \\
\text { desfavorable }\end{array}$ \\
02 & Media mañana & 1 & $\begin{array}{l}\text { Respuesta medianamente favorable } \\
03\end{array}$ \\
04 & Un día & 2 & Respuesta favorable \\
\hline
\end{tabular}

Respecto al tipo de interés que ofrece el banco en comparación con la banca privada es menor y con más ventajas, se tiene una respuesta favorable.

Tabla 5.

\begin{tabular}{cccl}
\multicolumn{1}{l}{ El tipo de interés que ofrece el banco en comparación con la banca privada } \\
\hline $\mathrm{N}^{\circ}$ & Alternativas & Puntaje & \multicolumn{1}{c}{ Sustento } \\
\hline 01 & $\mathrm{SI}$ & 2 & Respuesta favorable \\
02 & NO & 0 & Respuesta desfavorable \\
03 & No Sabe & 1 & Respuesta medianamente favorable \\
\hline
\end{tabular}

Respecto al canal de atención que realiza con frecuencia con su banco privado, se tiene alto puntaje en ventanillas, cajero automático e Internet.

Tabla 6.
\begin{tabular}{cccc}
\multicolumn{4}{c}{ Canales de atención que utiliza con frecuencia para sus operaciones } \\
\hline $\mathrm{N}^{\circ}$ & Alternativas & Puntaje & Sustento \\
\hline 01 & Ventanilla & 2 & Respuesta favorable \\
02 & Cajero Automático & 2 & Respuesta favorable \\
03 & Internet & 1 & Respuesta medianamente favorable \\
04 & Otros & 1 & Respuesta medianamente favorable \\
\hline
\end{tabular}

Respecto al conocimiento de la red de cajeros automáticos y ventanillas más grandes del Perú que la banca privada, hay una puntuación favorable.

Tabla 7.

Conocimiento sobre la red de cajeros automáticos y ventanillas

\begin{tabular}{cccl}
\hline $\mathrm{N}^{\circ}$ & Alternativas & Puntaje & \multicolumn{1}{c}{ Sustento } \\
\hline 01 & $\mathrm{SI}$ & 2 & Respuesta favorable \\
02 & NO & 0 & Respuesta desfavorable \\
03 & No Sabe & 1 & Respuesta medianamente favorable \\
\hline
\end{tabular}

Respecto al interés de los productos, tiene una puntuación alta los préstamos, seguido de tele giros y ahorros.

\begin{tabular}{lccl}
$\begin{array}{l}\text { Tabla } 8 . \\
\text { Interés de los productos que ofrece el banco }\end{array}$ & & \\
\hline $\mathrm{N}^{\circ}$ & Alternativas & Puntaje & Sustento \\
\hline 01 & Préstamo & 2 & Respuesta favorable \\
02 & Tele Giro & 1 & Respuesta medianamente favorable \\
03 & Ahorros & 1 & Respuesta medianamente favorable \\
04 & C. hipotecario & 1 & Respuesta medianamente favorable \\
\hline
\end{tabular}

Se tuvo como variables independientes los convenios con el sector privado medido con cantidad de convenios firmados y como variable dependiente la ampliación de colocaciones de préstamo, teniendo como indicador la cantidad de colocaciones realizadas a los empleados de las empresas privadas con las cuales se firmó los convenios. 


\section{Resultados y discusión}

Según la evaluación sobre la bancarización pública como factor explicativo del crecimiento regional, se encontró que, tanto en el análisis global como en el estudio de caso, existe una causalidad positiva del programa de crédito del banco sobre la variable consumo (medida indirectamente con la recaudación del IGV y con un menor éxito en el caso del empleo). La evaluación no sólo consideró la estimación puntual, sino también mostrar si el valor cero de los parámetros estimados podría ser considerado. En ningún caso se observó un valor estadísticamente igual a cero. Sin embargo, en la evaluación del modelo de datos de panel si se obtuvo parámetros con un valor negativo que podría estar vinculado a un problema de medición de la variable empleo.

El nuevo Préstamo orientado al Sector Privado tiene características diferentes por lo que, se deberá mitigar dichos riesgos potenciales tomando en consideración controles para el cobro o morosidad de los clientes que accederán a dicho producto. El Programa préstamos para el sector privado, estaría orientado a financiar un negocio, un viaje, el pago de estudios, una vivienda, la refacción y ampliación de la vivienda, entre otros propósitos.

La calidad crediticia del portafolio es saludable, así lo revelan los bajos niveles de morosidad y las provisiones cubren holgadamente la cartera vencida y judicial. Sin embargo, para el sector privado, el portafolio podría verse incrementado la morosidad a pesar que exista convenios entre el banco y la empresa; es por ello, que se refuerza la recuperación, en este caso por medio de empresas privadas dedicada al cobro de préstamos. Se puede observar las ventajas competitivas que adquiriría el banco frente a las posibilidades de aumentar su presencia en el mercado a través del impulso de programas como los préstamos con inclusión social.

Con la aplicación de los puntajes a los resultados de la encuesta realizada, se puede obtener la siguiente tabla:

\begin{tabular}{lc} 
Tabla 9. \\
Puntajes a los resultados \\
\hline Puntaje & Frecuencia \\
\hline$[2-4>$ & 14 \\
{$[4-6>$} & 64 \\
{$[6-8>$} & 59 \\
{$[8-10>$} & 110 \\
{$[10-12>$} & 104 \\
{$[12-14]$} & 19 \\
\hline Total & 370 \\
\hline
\end{tabular}

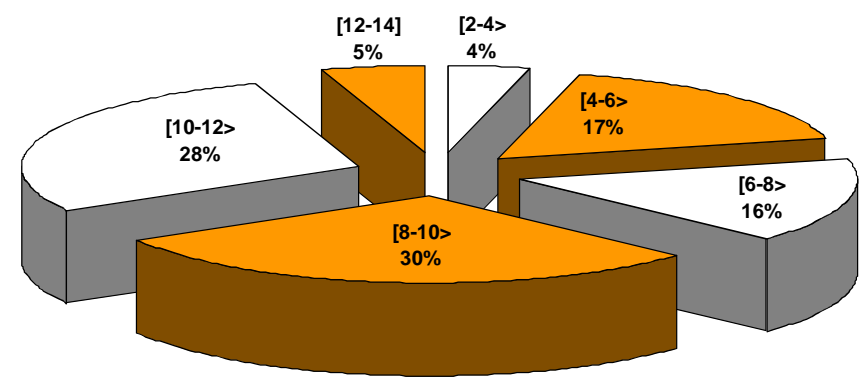

Figura 1. Resultados calidad de portafolio

La puntuación máxima que se podría obtener es 14 puntos, lo que representa el máximo grado de posibilidad que, incrementando los convenios del banco con el sector privado, se incrementarán los préstamos. La puntuación mínima que se podría obtener es 2 puntos, lo que representa el mínimo grado de posibilidad que, incrementando los convenios del banco con el sector privado, se incrementarán los préstamos.

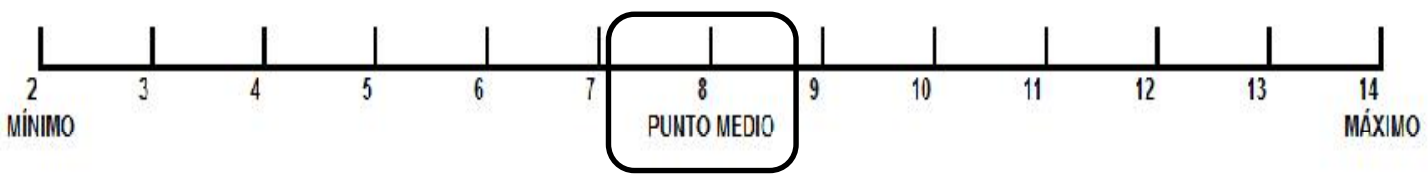

Figura 2. Puntuación mínima

$\mathrm{HO}: \mathrm{u}=8$, incrementando los convenios del banco con el sector privado, no se incrementarán los préstamos en los lugares del Perú, donde la institución es única oferta bancaria.

H1: u > 8, incrementando los convenios del banco con el sector privado, se incrementarán los préstamos en los lugares del Perú, donde la institución es única oferta bancaria. 
Tabla 10

Tabla de frecuencia

\begin{tabular}{lllll}
\hline Puntaje & Punto medio & Frecuencia $(\mathrm{x})$ & Resultado $\left(\mathrm{f}_{\mathrm{x}}\right)$ & Media $\left(\mathrm{x}_{1}\right)$ \\
\hline$[2-4>$ & 3 & 14 & 42 \\
{$[4-6>$} & 5 & 64 & 320 & 3156 \\
{$[6-8>$} & 7 & 59 & 413 & 370 \\
{$[8-10>$} & 9 & 110 & 990 & $=8.53$ \\
{$[10-12>$} & 11 & 104 & 1144 & 247 \\
{$[12-14]$} & 13 & 19 & 3156 & \\
TOTAL & & 370 &
\end{tabular}

Dado a que el valor $Z$ calculado no queda en la región comprendida entre -1.96 y +1.96, entonces rechazamos la hipótesis nula (HO: Incrementando los convenios del banco con el sector privado, no se incrementarán los préstamos en los lugares del Perú donde la institución es única oferta bancaria) y aceptamos la hipótesis alternativa (H1: Incrementando los convenios del banco con el sector privado, se incrementarán los préstamos en los lugares del Perú, donde la institución es única oferta bancaria)

\section{Discusión}

La propuesta es una gran medida de fomento de inclusión social, que podría considerarse como elemento fundamental en los futuros programas, productos y servicios que se ofrezcan en el mercado.

Por otro lado, de evidenciarse que el producto llamado Programa de Préstamos del banco contara con estándares de eficiencia surgido bajo la modalidad específica de privatización desarrollada, se comprobaría la tesis planteada y corroborada en algunos estudios específicos de la no necesidad de transferencia de la propiedad de una empresa pública para que ésta pueda presentar estándares de eficiencia comparados con la empresa privada.

La comprobación de un mayor nivel de eficiencia en el uso de los recursos ahorrados de forma descentralizada al servir de base de financiamiento del Programa de Préstamos, podría también servir como un elemento a considerar para la elaboración de políticas públicas relacionadas a la descentralización económica.

Respecto al crecimiento de la productividad y el desarrollo económico estamos de acuerdo con Levine et al (2003a) señalando que los mejores sistemas financieros aumentan la probabilidad de que surjan innovaciones exitosas y por consiguiente aceleran el crecimiento económico. Además, También consideramos que el sistema financiero es un indicador de desarrollo basado en ratios de crédito al Sector Privado como lo sostienen Calderón y Schmidt-Hebbel (2013). 


\section{Conclusiones}

El banco puede aumentar su presencia en el mercado a través del impulso de programas como los préstamos con inclusión social, mediante la definición de requerimientos específicos podría lograrse el éxito de los programas de inclusión social, fortaleciendo en las instituciones el trabajo coordinado enfocado al cliente para establecer y lograr el cumplimiento de la política de calidad y los objetivos de calidad, generando consistentemente productos y servicios que satisfagan las necesidades y expectativas de sus clientes.

Debido a la modalidad de préstamos que otorga el banco referido al sector público, la calidad crediticia del portafolio es saludable, así lo revelan los bajos niveles de morosidad y las provisiones cubren holgadamente la cartera vencida y judicial. Sin embargo, para el sector privado, el portafolio podría verse incrementado la morosidad a pesar que exista convenios entre el banco y la empresa; es por ello, que se refuerza la recuperación, en este caso por medio de empresas privadas dedicada al cobro de préstamos.

Para que los nuevos clientes que vienen del sector privado puedan acceder a este producto, previamente deberá existir el convenio entre la empresa privada con el banco y estar ubicada en los sectores donde el banco es la única oferta bancaria. El Programa préstamos para el sector privado, puede beneficiar para que nuestros clientes pueden tener la facilidad de financiar el negocio propio, el viaje anhelado, el pago de estudios, el sueño de la casa propia, la refacción y ampliación de la vivienda, entre otros propósitos.

\section{Referencias bibliográfic as}

Aguilar, G., Camargo, G. y Morales, R. (2004): Análisis de la Morosidad en el Sistema Bancario Peruano: Informe final de investigación; Instituto de Estudios Peruanos. Octubre.

Informe especial disponible en www.bcrp.gob.pe

Banco Central de Reserva del Perú: Memoria (varios años).

Banco Central de Reserva del Perú, Nota Semanal, Series Estadísticas en www.bcrp.gob.pe.

Blyde,j., y Fernandez Arias (2004) Why Does Latin America Grow More Slowly? In Sources of Growth in Latin America, editado por Eduardo Fernandez-Arias, Rody Manuelli, and Juan S. Blyde. Washington. IDB.

Calderon,C., y Serven L.,(2003) Macroeconomic Dimensions of Infraestructura in Latin America. Washington. World Bank.

Caskey J.(2004) Organizing Acces to Capital: Advocacy and the Democratization of Principal Institutions. Journal of Urban Studies. Vol 41 Iss 13

Claessens, S. y L. Laeven (2004) What Drives Bank Competition? Some International Evidence. Journal of Money, Credit and Banking. Vol. 36 №2. June, Part 2, pp. $563-583$

Levine R.Demirguc-Kunt A y Beck Thorsten (2003). Bank Concentration, Competition and Crises: First Results. Journal of Banking \&Finance. Vol. 30 Iss 5 pp 1581 - 1603

Mimeo Julio Levine R, Demirguc-KuntA y Beck Thorsten 2006 Bank Supervision and Corruption in Lending. Journal of Monetary Economics. Vol 53 Iss. 8 pp 2132- 2163.

Rebolledo P. y Soto R. 2004. Estructura del mercado de crédito y las tasas de interés. Estudios Económicos BCRP 11. Octubre.

Superintendencia de Banca y Seguros del Perú. Estadísticas del Sistema bancario y financiero. En www.sbs.gob.pe 